\title{
AN ENGEL CONDITION WITH DERIVATION
}

\author{
CHARLES LANSKI
}

(Communicated by Maurice Auslander)

\begin{abstract}
Let $R$ be a prime ring, $L$ a noncommutative Lie ideal of $R$, and $D$ a nonzero derivation of $R$. If for each $x \in L,[D(x), x]_{k}=$ $[[\cdots[D(x), x], x], \ldots, x]=0$ with $k$ fixed, then $\operatorname{char}(R)=2$ and $R \subseteq$ $M_{2}(F)$ for $F$ a field.
\end{abstract}

In a recent paper, Vukman [12] gives an elementary but lengthy calculation that yields extensions of a well-known theorem of Posner [10] on centralizing derivations of prime rings. The purpose of this note is to show that by using the theory of differential identities one can fairly quickly generalize the results of Vukman to higher commutators, eliminate his restriction on characteristic, and extend the results from prime rings to Lie ideals in prime rings.

The theory of differential identities was formally initiated by Kharchenko [5]. Its use here will not be explicit and arises only in reference to results in subsequent work of Chuang [1] and the author [6]. The one related object we need to mention is the symmetric quotient ring, introduced in [5] and required in [1] and [6] (see [9] for some details). Henceforth, we let $R$ denote a prime ring with extended centroid $C$ and symmetric quotient ring $Q$. All that we need here about these objects is that $R \subseteq Q, Q$ is a prime ring whose center is the field $C$, and that $C$ is the centralizer of $R$ in $Q$. By $D$ we always mean a nonzero derivation of $R$, and $D=\operatorname{ad}(A)$ for $A \in Q$ implies that $D(r)=[A, r]=A r-r A$.

In [10] Posner proved that $R$ must be commutative if $[D(x), x]$ is central for any $x \in R$. Set $[y, x]_{1}=[y, x]=y x-x y$ for any $x, y \in R$, and for $k>1$ let $[y, x]_{k}=\left[[y, x]_{k-1}, x\right]$. The results of Vukman [12] show that $R$ is commutative if either $[D(x), x]_{2}=0$ for all $x \in R$ and $\operatorname{char}(R) \neq 2$ or if $[D(x), x]_{2}$ is central for all $x \in R$ and $\operatorname{char}(R) \neq 2,3$. We consider the more general Engel condition when for a fixed $k>0,[D(x), x]_{k}=0$ for all $x \in L$, a noncommutative Lie ideal of $R$. Our first theorem will give the result for ideals. It incorporates the arguments needed for Lie ideals but avoids some technical complications of that case.

Two well-known observations are crucial to our arguments and for convenience we state them as lemmas.

Received by the editors January 8, 1991 and, in revised form, November 11, 1991.

1991 Mathematics Subject Classification. Primary 16W10; Secondary 16W25, 16N60, 16U80. 
Lemma 1. If $R$ is not commutative and satisfies a polynomial identity, then for some field $F, R \subseteq M_{n}(F)$ with $n>1$ and $M_{n}(F)$ satisfies the same identity. Proof. This standard fact follows from [4, Theorem 2, p. 57 and Lemma 1, p. 89].

Lemma 2. Let $R$ be a noncommutative simple algebra, finite-dimensional over its center $Z$. If $g\left(x_{1}, \ldots, x_{t}\right) \in R *_{Z} Z\left\{x_{j}\right\}$, the free product over $Z$, is an identity for $R$ that is homogeneous in $\left\{x_{1}, \ldots, x_{t}\right\}$ of degree $d$, then for some field $F$ and $n>1, R \subseteq M_{n}(F)$ and $g\left(x_{1}, \ldots, x_{t}\right)$ is an identity for $M_{n}(F)$.

Proof. This is essentially [11, Theorem 2.3.29, p. 131], at least when $g\left(x_{1}, \ldots, x_{t}\right)$ is a polynomial identity. We provide a short argument that will be needed later. If $Z$ is finite then $R=M_{n}(F)$, and there is nothing more to prove. When $Z$ is infinite, then $M_{n}(F)=R \otimes_{Z} F$ for $F$ a splitting field of $R$, and standard arguments show that $R \otimes F$ satisfies $g\left(x_{i}\right)$. Specifically, consider $R\left[c_{j}\right]$ for $\left\{c_{j}\right\}$ commuting indeterminates over $R$, and set $Y_{i}=\sum_{j=1}^{d} r_{i j} c_{j} \in R\left[c_{j}\right]$. Then $g\left(Y_{1}, \ldots, Y_{t}\right)=\sum m_{w}\left(c_{j}\right) g_{w}\left(r_{i j}\right)$, where $\left\{m_{w}\left(c_{j}\right)\right\}$ are all monomials of degree $d$ in $\left\{c_{j}\right\}$. When $z_{j} \in Z$ replaces $c_{j}$, then $Y_{i} \in R$ so $g\left(Y_{1}, \ldots, Y_{t}\right)=0$ and a Vandermonde determinant argument shows that each $g_{w}\left(r_{i j}\right)=0$. It follows that $g\left(x_{1}, \ldots, x_{t}\right)$ is an identity for $R \otimes K$ for any extension field $K$ of $Z$.

We can now prove our first theorem.

Theorem 1. If $I$ is a nonzero ideal of $R$ so that $[D(r), r]_{k}=0$ for all $r \in I$ and $k>0$ fixed, then $R$ is commutative.

Proof. The formal expression $[D(x), x]_{k}$ is an identity with derivation satisfied by $I$, so by [1, Main Theorem, p. 251 and Remark 1, p. 278], either $[y, x]_{k}$ is an identity for $R$ or $D=\operatorname{ad}(A)$ for $A \in Q$. In the first case $R$ satisfies the polynomial identity $[y, x]_{k}$, so we may apply Lemma 1 and conclude that $R$ is commutative or that $M_{n}(F)$ satisfies $[y, x]_{k}$ with $n>1$. But in $M_{n}(F)$, $\left[e_{21}, e_{11}\right]_{k}=e_{21} \neq 0$, so $R$ must be commutative. Therefore, we may assume that $D=\operatorname{ad}(A)$ and that $I$ satisfies $g(x)=[A, x]_{k+1}$. At this point we could apply a major result of Chuang and Lin [2] to obtain the contradiction $A \in C$, and so $D=0$. However, the argument here is not difficult and is needed for the Lie ideal case, so we proceed with it.

Since $I$ satisfies $g(x)$, it follows that $R C$ is a primitive ring with $H=$ $\operatorname{soc}(R C) \neq 0$ and $e H e$ is finite dimensional over $C$ for any minimal idempotent $e \in R C$ [6, Proposition, p. 769; 8, Theorem 2, p. 587]. We may assume that $H$ is not commutative, since otherwise $R$ is commutative, finishing the proof. We claim that $H$ satisfies $g(x)$. If $C$ is finite then $J C \subseteq I$ for some nonzero ideal $J$ of $R$, and since $H$ is the minimal ideal in $R C, H \subseteq J C \subseteq I$ does satisfy $g(x)$. When $C$ is infinite the proof of Lemma 2 works: in that argument take a large collection of $\left\{z_{j}\right\} \subseteq C$ and an ideal $J \subseteq I$ of $R$ with $J z_{j} \subseteq I$ for all $z_{j}$; then using all $r_{i j} \in J$ shows that $H \subseteq J C$ satisfies $g(x)$.

Clearly, $H$ satisfies $[A, x]_{m}$ for $m>k$, and so, we may assume that $m$ is odd. But now for $e=e^{2} \in H, 0=[A, e]_{m}=[A, e]$, so $A$ commutes with all idempotents in $H$. Since $H$ is a simple ring, either $H$ is generated by its idempotents [3, Corollary, p. 18 and Corollary, p. 9], giving the contradiction $A \in C$, or else $H$ contains no nontrivial idempotent. In the latter case, $H$ is 
a finite-dimensional division algebra over $C, H=R C=Q$, and so, $A \in H$. By Lemma 2, $[A, x]_{m}$ is satisfied by some $M_{n}(F)$, and the argument just above shows that $A$ commutes with all idempotents in $M_{n}(F)$, again giving the contradiction $A \in C$, and completing the proof of the theorem.

Before stating our main result, we recall that an additive subgroup $L$ of $R$ is a Lie ideal if $[x, r] \in L$ for all $x \in L$ and all $r \in R$. A well-known and important fact about noncommutative Lie ideals is that any such contains [ $M, M]$ for a nonzero ideal $M$ of $R$. This follows from the computations in [3, Chapter 1] and is explicitly given as [7, Lemma 2, p. 280].

Theorem 2. Let $L$ be a noncommutative Lie ideal of $R$. If $[D(x), x]_{k}=0$ for all $x \in L$ and $k>0$ fixed, then $\operatorname{char}(R)=2$ and $R \subseteq M_{2}(F)$ for $F$ a field, so $[D(x), x]_{2}=0$.

Proof. We follow the outline of the proof of Theorem 1. Note that $R$ is not commutative by the assumption on $L$. Since $[M, M] \subseteq L$ for $M$ a nonzero ideal of $R$, we have $[D([x, y]),[x, y]]_{k}=0$ for all $x, y \subseteq M$, and it follows that $[[D(x), y],[x, y]]_{k}+[[x, D(y)],[x, y]]_{k}=0$. If $D \neq \operatorname{ad}(A)$ for $A \in Q$, then by [1, Main Theorem, p. 251 and Remark 1, p. 278], we may conclude that $[[t, y],[x, y]]_{k}$ is an identity for $R$, so Lemma 1 shows that this is also an identity for $M_{n}(F)$ with $n>1$ and $R \subseteq M_{n}(F)$. However, if $\operatorname{char}(R) \neq 2$ then for $t=e_{11}, y=e_{12}$, and $x=e_{21}$, [[t,y], $\left.[x, y]\right]_{k}=2^{k} e_{12} \neq 0$, and if $n>2$ then for $t=e_{23}, y=e_{31}$, and $x=e_{13},[[t, y],[x, y]]_{k}=e_{21} \neq 0$. Consequently, when $D \neq \operatorname{ad}(A)$, we must have char $(R)=2$ and $R \subseteq M_{2}(F)$. Squares of commutators are central in $M_{2}(F)$, so in this case, $[D(x), x]_{2}=$ $\left[D(x), x^{2}\right]=0$ for all $x \in L \subseteq\left[M_{n}(F), M_{n}(F)\right]$.

Now assume that $D=\operatorname{ad}(A)$, so $M$ satisfies the identity $[A,[x, y]]_{k+1}=0$. As in Theorem 1 , this identity is satisfied by $H=\operatorname{soc}(R C) \neq 0$. Suppose next that $H$ contains three orthogonal and minimal idempotents $e, f$, and $g$. Since $f H$ and $g H$ are isomorphic $H$ modules, there are $f b g, g a f \in H$ with $f b g a f=f$ and $g a f b g=g$, so $f-g=[f b g, g a f]$. Thus

$$
e A f=e[A,[f b g, g a f]]_{k+1} f=0
$$

for any orthogonal rank one idempotents $e$ and $f$. It follows easily that $[A, e]=0$ for any rank one idempotent, and since $H$ is generated by these idempotents, $[A, H]=0$ results. This gives the contradiction $A \in C$ and $D=0$, so $H$ cannot contain three orthogonal idempotents.

We may assume that $H=M_{2}(E)$ for $E$ a division algebra, finite-dimensional over its center, so $A \in Q=H$ as in Theorem 1. Using Lemma 2 shows that $H \subseteq M_{n}(F)$ and that $M_{n}(F)$ satisfies $[A,[x, y]]_{k+1}$. If $n>2$ then as we have just seen, $A$ commutes with all idempotents in $M_{n}(F)$, forcing the contradiction $D=0$. Therefore, $n=2$. Let $t=[x, y] \in\left[M_{2}(F), M_{2}(F)\right]$ with $t^{2}=z(t) \in F$, observe as in Theorem 1 that $[A, t]_{m}=0$ for $m=2 s+1$, and compute that $0=[A, t]_{m}=(4 z(t))^{s}[A, t]$. If $\operatorname{char}(F) \neq 2$, then since $A \notin F$ there is $t \in\left[M_{2}(F), M_{2}(F)\right]$ with $t^{2} \in F-(0)$ and $[A, t] \neq 0$. Specifically, if $t=e_{12}+e_{21}=\left[e_{11}, e_{12}-e_{21}\right]$, then $[A, t] \neq 0$ unless $A=$ $a I_{2}+b\left(e_{12}+e_{21}\right)$, in which case $[A, w] \neq 0$ for $w=e_{11}-e_{22}=\left[e_{12}, e_{21}\right]$. Consequently, we must have $\operatorname{char}(R)=2$, and since $R \subseteq H \subseteq M_{2}(F)$, the proof is complete. 


\section{REFERENCES}

1. C. L. Chuang, *-differential identities in prime rings with involution, Trans. Amer. Math. Soc. 316 (1989), 251-279.

2. C. L Chuang and J. S. Lin, On a conjecture by Herstein, J. Algebra 126 (1989), 119-138.

3. I. N. Herstein, Topics in ring theory, The Univ. of Chicago Press, Chicago, 1969.

4. N. Jacobson, PI-algebras, Lecture Notes in Math., vol. 441, Springer-Verlag, New York, 1975.

5. V. K. Kharchenko, Differential identities of prime rings, Algebra and Logic 17 (1978), 155168.

6. C. Lanski, Differential identities in prime rings with involution, Trans. Amer. Math. Soc. 291 (1985), 765-787.

7. ___ Differential identities, Lie ideals, and Posner's theorems, Pacific J. Math. 134 (1988), 275-297.

8. W. S. Martindale III, Prime rings satisfying a generalized polynomial identity, J. Algebra 12 (1969), 576-584.

9. D. Passman, Infinite crossed products, Pure Appl. Math., vol. 135, Academic Press, New York, 1989.

10. E. C. Posner, Derivations in prime rings, Proc. Amer. Math. Soc. 8 (1957), 1093-1100.

11. L. Rowen, Polynomial identities in ring theory, Pure Appl. Math., vol. 84, Academic Press, New York, 1980.

12. J. Vukman, Commutating and centralizing mappings in prime rings, Proc. Amer. Math. Soc. 109 (1990), 47-52.

Department of Mathematics, University of Southern California, los Angeles, CALIFORNia 90089-1113 